\title{
Characterization and Validation of a Novel Equilibrium Passive Sampler for Prediction of Porewater Methylmercury
}

SPENCER WASHBURN ${ }^{1 *}$, UPAL GHOSH ${ }^{2}$, JADA DAMOND ${ }^{2}$, AND CYNTHIA GILMOUR ${ }^{1}$

${ }^{1}$ Smithsonian Environmental Research Center, MD 21037 , USA (*Correspondence washburns@si.edu; gilmourc@si.edu)

${ }^{2}$ Department of Chemical, Biochemical, and Environmental Engineering, University of Maryland Baltimore County, MD 21250, USA (ughosh@umbc.edu;

damond1@umbc.edu)

Accurate direct measurement of aqueous methylmercury $(\mathrm{MeHg})$ - especially in sediment porewaters - is challenging. As such, there remains a need for a simple, standardized passive sampling system from which porewater $\mathrm{MeHg}$ concentrations and $\mathrm{MeHg}$ bioavailability can be calculated. This study details the validation of a novel material comprised of agarose gel with embedded activated carbon particles $(\mathrm{ag}+\mathrm{AC})$ as an equilibrium passive sampler capable of estimating porewater $\mathrm{MeHg}$ concentrations through laboratory experiments in a combination of idealized media and realistic sediment microcosms.

Isotherm bottle experiments with ag+AC polymers were conducted to constrain partitioning of various environmentlaly relevant $\mathrm{MeHg}$ species to these materials. Partitioning coeffeicients ranged from $\log \left(\mathrm{K}_{\mathrm{ps}}\right)=2.83$ for $\mathrm{MeHgDOM}$ to $\log \left(\mathrm{K}_{\mathrm{ps}}\right)=3.03$ for MeHgCys. To demonstrate equilibrium exchange of an environmentally relevant $\mathrm{MeHg}$ species, a dual label loading/desoprtion experiment was conducted. After equilibration with $\mathrm{Me}^{199} \mathrm{HgDOM}$ in solution, ag+ $\mathrm{AC}$ polymers were transferred to solutions containing $\mathrm{Me}^{198} \mathrm{HgDOM}$. Both complexes approached equilibrium with the samplers after 14 days while maintiaining mass balance. This study demonstrated reversible exchange of MeHgDOM species, providing further evidence the ag+AC polymer material is capable of equilibrium measurements of environmentally-relevant $\mathrm{MeHg}$ species within a reasonable time frame.

Sampler deployment across the sediment-water interface of sediment microcosms provided estimatess of measured surface water and porewater $\mathrm{MeHg}$ concentrations within a factor of 2, based on the measured $\mathrm{K}_{\mathrm{ps}}$ for MeHgDOM. This result suggests that the samplers can be used to evaluate aqueous MeHg gradients with depth in sediments, and that a standardized $\mathrm{K}_{\mathrm{ps}}$ based on model complexes can be used for reasonably accurate predictions across many sites. 